\title{
BMJ Open Out-of-sequence DTP and measles vaccinations and child mortality in Guinea-Bissau: a reanalysis
}

\author{
Sanne M Thysen, ${ }^{\oplus, 2,3,4}$ Amabelia Rodrigues, ${ }^{2}$ Peter Aaby, ${ }^{2,3}$ Ane B Fisker ${ }^{1,2,3}$
}

To cite: Thysen SM, Rodrigues A, Aaby P, et al. Out-of-sequence DTP and measles vaccinations and child mortality in GuineaBissau: a reanalysis. BMJ Open 2019:9:e024893. doi:10.1136/ bmjopen-2018-024893

- Prepublication history and additional material for this paper are available online. To view these files, please visit the journal online (http://dx.doi. org/10.1136/bmjopen-2018024893).

Received 22 June 2018 Revised 06 August 2019 Accepted 09 August 2019
Check for updates

(C) Author(s) (or their employer(s)) 2019. Re-use permitted under CC BY-NC. No commercial re-use. See rights and permissions. Published by BMJ.

${ }^{1}$ OPEN, Institute of Clinical Research, University of Southern Denmark, Odense, Denmark ${ }^{2}$ Bandim Health Project, INDEPTH Network, Bissau, Guinea-Bissau

${ }^{3}$ Research Centre for Vitamins and Vaccines, Bandim Health Project, Statens Serum Institut, Copenhagen, Denmark

${ }^{4}$ Center for Global Health (GloHAU), Aarhus University, Aarhus, Denmark

Correspondence to Dr Sanne M Thysen; s.thysen@bandim.org

\begin{abstract}
Objectives To assess whether the sequence of diphtheria-tetanus-pertussis vaccine (DTP) and measles vaccine (MV) was associated with child survival in a dataset previously used to assess non-specific effects of vaccines with no consideration of vaccination sequence. Design Prospective cohort study analysed using the landmark approach.
\end{abstract}

Setting Bandim Health Project's Health and Demographic Surveillance System covering 100 village clusters in rural Guinea-Bissau. The recommended vaccination schedule was BCG and oral polio vaccine (OPV) at birth, DTP and OPV at 6, 10 and 14 weeks, MV at 9 months and booster DTP and OPV at 18 months of age.

Participants Children aged 9-17 months (main analysis) and 18-35 months (secondary analysis: age of booster DTP) with vaccination status assessed between April 1991 and April 1996.

Methods Survival during the 6 months after assessing vaccination status was compared by vaccination sequence in Cox-proportional hazards models with age as underlying time. Analyses were stratified by sex and village cluster. Main outcome measure Mortality rate ratio (MRR) for out-of-sequence vaccinations compared with in-sequence vaccinations.

Results Among children aged 9-17 months, 60\% of observations (3574/5937) were from children who had received both MV and DTP. Among these, 1590 observations were classified as in-sequence vaccinations (last DTP before MV), and 1984 observations were out-of-sequence vaccinations (1491: MV with DTP and 493: MV before DTP). Out-of-sequence vaccinations were associated with higher mortality than in-sequence vaccinations (MRR 2.10, 95\% Cl 1.07 to 4.11); the MRR was $2.30(95 \% \mathrm{Cl} 1.15$ to 4.58$)$ for MV with DTP and $1.45(95 \% \mathrm{Cl} 0.50$ to 4.22$)$ for DTP after MV. Associations were similar for boys and girls $(p=0.77)$. Between 18 and 35 months the mortality rate increased among children vaccinated in-sequence and the differential effect of outof-sequence vaccinations disappeared.

Conclusion Out-of-sequence vaccinations may increase child mortality. Hence, sequence of vaccinations should be considered when planning vaccination programmes or introducing new vaccines into the current vaccination schedule.

\section{INTRODUCTION}

Child mortality has declined significantly between 2000 and 2015. ${ }^{1}$ Part of this decline
Strengths and limitations of this study

Vaccination status of the children were only updated at the inspection of a vaccination card.

- This study used the landmark analyses and thus prevented survival bias.

- Misclassification of vaccinations due to the landmark approach would yield conservative estimates.

- Booster doses of diphtheria-tetanus-pertussis (DTP) were not registered before 1996, and we were therefore not able to make any firm conclusions of the effect of booster DTP.

- Sensitivity analyses were conducted to limit the effect of vaccinations during follow-up.

is due to a reduction in preventable childhood diseases much of which is commonly ascribed to vaccines. ${ }^{2}$ Vaccines are designed to protect against specific pathogens. ${ }^{3}$ However, vaccines may have broader effects aside from the disease-specific protection with the live vaccines stimulating the immune system and reducing mortality by more than can be explained by preventing the target infection. ${ }^{4-7}$ Hence, due to beneficial non-specific effects (NSEs) of live vaccines, vaccines may have played an even larger role in the decline of childhood mortality than usually assumed.

Studies from the introduction of the measles vaccine (MV) in the 1970s and 1980s from Asia and Africa showed larger reductions in mortality than could be ascribed to the prevention of measles infection. ${ }^{8-10}$ Both observational studies and randomised controlled trials (RCTs) have later confirmed lower mortality among measles-vaccinated children compared with measles-unvaccinated children. ${ }^{11-13}$ Based on accumulating evidence, WHO's Strategic Advisory Group of Experts on immunisation recently reviewed the evidence for NSEs of some vaccines, and concluded that the evidence for MV was consistent with beneficial NSEs, especially for girls. $^{714}$ 
The introduction of diphtheria-tetanus-pertussis vaccine (DTP) in the 1980s was associated with higher overall mortality, despite the protection against the specific diseases. ${ }^{15-17}$ Other studies comparing mortality of DTP-vaccinated children and DTP-unvaccinated children have later confirmed the negative NSEs, especially for girls. ${ }^{11-21}$ The WHO review of NSEs stated that beneficial or deleterious NSEs of DTP could not be confirmed nor refuted based on the evidence available. ${ }^{714}$ However, the WHO review included studies with major survival bias; if the meta-analysis is restricted to studies with documentation of vaccination status and prospective follow-up, DTP-vaccinated children had twofold higher mortality than DTP-unvaccinated children. ${ }^{22}$

Both observational studies ${ }^{182023-27}$ and RCTs ${ }^{1928}$ suggest that the NSEs depends most strongly on the most recent vaccination and that sequence of vaccinations therefore is important. RCTs have compared inactivated vaccine after medium-titre or high-titre MV with standard-titre MV after inactivated vaccine. A meta-analysis of the trials indicates that receiving an inactivated vaccine after a live MV was associated with a mortality rate ratio (MRR) of 1.38 (95\% CI 1.05 to 1.83 ) compared with receiving live MV after an inactivated vaccine, the negative effect being particularly strong for females. ${ }^{28}$

In the first study that assessed the effect on mortality of MV and DTP, having received MV versus no MV was associated with an MRR of 0.48 (95\% CI 0.27 to 0.87 ); in contrast, having received DTP versus no DTP was associated with higher mortality $(\mathrm{MRR}=1.84,95 \%$ CI 1.10 to 3.10). ${ }^{11}$ The analysis did not consider sequence of vaccinations, the potential importance of which had not yet been detected. We took advantage of this historical datase $\mathrm{t}^{11}$ to test if the different sequences of DTP and MV vaccinations were associated with mortality. The issue is particularly important now because WHO is planning to add several non-live vaccines to the vaccination schedule, ${ }^{29}$ including booster DTP and RTS,S malaria vaccine, and some will be given after MV.

\section{METHODS \\ Setting}

Data were collected within the Bandim Health Project's Health and Demographic Surveillance System (HDSS) in rural Guinea-Bissau. The HDSS was established in 1990 using the Expanded Programme on Immunizations methodology, randomly selecting 20 clusters of 100 women in each of the five largest health regions. Women of fertile age and their children below 5 years of age were followed through biannual visits. Women were registered at 14-16 years of age or when they moved into the village and were followed to death or migration. Newly registered women were interviewed about their past obstetric history, age, ethnicity and whether they had attended school. Children were registered during pregnancy or when they moved into the village. Children were followed until death, migration or 5 years of age.
At all visits, vaccination status, nutritional status and vital status were assessed. Vaccination status was assessed by inspection of a vaccination card. Children with no vaccination card and whose mother stated that the child had never received any vaccine were considered 'unvaccinated'. Only children with ascertained vaccination status (seen vaccination card, confirmed unvaccinated) were included in the analyses. Nutritional status was assessed by measurement of the child's mid-upper arm circumference (MUAC).

\section{Vaccination programme and definition of exposure}

The vaccination schedule consisted of BCG vaccine and oral polio vaccine (OPV) at birth, 3 doses of DTP and OPV at 6,10 and 14 weeks of age, MV at 9 months of age and booster doses of DTP and OPV at 18 months of age. The vaccination schedule did not change during the study period. Vaccinations were provided through the national immunisation programme. Systematic registration of DTP and OPV booster doses were only initiated in 1996, and thus, booster doses were not registered during the study period.

Children were divided into five groups according to the most recent vaccination(s) at the time their vaccination card was inspected: one group consisted of children, who were vaccinated in the recommended sequence, having received MV after DTP (DTP $<\mathrm{MV})$. Two groups were vaccinated out-of-sequence: children who had received DTP and MV simultaneously (DTP=MV), and children who had received DTP after MV (DTP $>$ MV). Two groups had not received MV; children who had received DTP, but had not received MV (DTP, no MV) and children who had neither received MV nor DTP (no DTP, no MV).

\section{Study population}

Children aged 9-35 months when visited between 9 April 1991 and 24 April 1996 were eligible for the study. Figure 1 depicts the combined mortality rate of all study children. Mortality declines with age as expected in the beginning, but around 21 months of age the mortality rate increases. The primary analysis is the age group 9-17 months since this is the period after MV is scheduled and before the scheduled age of booster dose of DTP. Children aged 18-35 months at the time of visit were included in a secondary analysis since they could have received a booster dose of DTP after their in-sequence or out-of-sequence vaccinations.

\section{Statistical analyses}

Baseline characteristics for different vaccination groups were compared using $\chi^{2}$ test, Kruskal-Wallis rank test and one-way analysis of variance comparison. We also compared baseline characteristics of children included in the analyses with children registered in the HDSS, but not included in the analyses using $\chi^{2}$ test, t-test and Wilcoxon rank-sum test. MUAC of children was expressed as a z-score compared with the 2006 WHO growth reference, ${ }^{30}$ thus obtaining a standardised measure. 


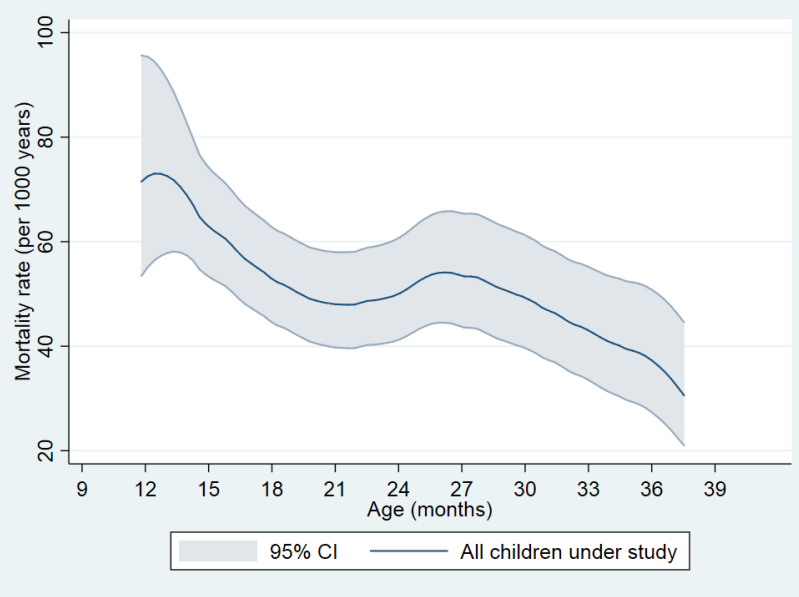

Figure 1 Overall mortality rate among children visited between 9 and 35 months of age. The figure plots the unadjusted mortality rates for children with a vaccination card seen between 9 and 35 months of age. The smoothed mortality curve starts shortly before 12 months of age, where the first event occurs. Only few children contribute observation time between 9 and 12 months.

Using a Cox proportional hazards model with age as underlying timescale, we compared mortality rates of children vaccinated out-of-sequence and children missing MV with the mortality rates of children vaccinated in-sequence. Children entered the analysis at the date of inspection of the vaccination card and remained in the analysis in the same vaccination group until the subsequent village visit, 6 months after the visit, death or migration, whichever came first. A child could therefore contribute with two non-overlapping periods if the vaccination status was assessed at more than one visit within the relevant age range (9-17 months). The booster doses of DTP and OPV administered at 18 months of age was not registered consistently and we were therefore not able to account for which children had received the booster doses; we therefore censored at 18 months of age in the main analysis.

The data were analysed using the landmark approach, ${ }^{31}$ in which the child's vaccination status is only updated when the vaccination status is re-assessed at the next home visit. If we had used the actual vaccination dates obtained at subsequent home visits to change the vaccine status, we would have better vaccination information for children who survived and had kept their vaccination cards, whereas the families of children who died between visits were likely to have discarded the vaccination card. As a consequence, the survivors would be given risk-free survival time for their new vaccination status, whereas it would not be known if the dead child had been vaccinated, and the child would therefore be misclassified as less vaccinated or unvaccinated. Such 'risk-free' survival time will strongly inflate the estimated benefit of the last vaccination. To avoid such survival bias, we have therefore chosen the landmark approach. ${ }^{31}$
In a secondary analysis, we assessed the effects of out-of-sequence vaccinations among children who were eligible for the DTP booster dose. In this analysis, we included children aged 18-35 months at the time of visit.

Since previous studies have reported sex-differential NSEs, all analyses were stratified by sex and separate estimates by sex are presented. All analyses were stratified by village cluster, thus comparing only children from the same community. All available baseline characteristics (table 1) were included in the analyses one by one. No variable changed the main estimate by $>10 \%$ and adjusted estimates are therefore not presented.

The original study assessed the effect of MV compared with no MV. To account for sequence of vaccination, we reanalysed the NSEs of MV comparing children vaccinated in-sequence with MV after DTP with children with no MV (DTP, no MV and no DTP, no MV).

\section{Sensitivity analyses}

Since many children were vaccinated during follow-up, that is, after the inspection of their vaccination card, which allowed their exposure group to be classified, we conducted two sensitivity analyses to limit the effect of vaccines administered during follow-up. In the first sensitivity analysis, we censored observation time at 2 months after entry. In the second sensitivity analysis, we included only children who had completed three DTP vaccinations and were therefore not eligible for further doses during follow-up.

\section{Ethical considerations}

The data were derived from the HDSS routine data collection, which has been ongoing since 1990 in collaboration with the Ministry of Health in Guinea-Bissau. ${ }^{11}$

\section{Patient and public involvement}

The communities were involved in locating households, when the HDSS was setup and contributed information allowing tracing of internal migrants between villages throughout the study period. No participants were involved in setting the research question or the outcome measure, nor were they involved in developing plans for recruitment, design or implementation of the study. No participant was asked to advise on interpretation or writing up the results. The results are disseminated to the national public health institute. There are no plans to disseminate the results of the research to study participants or the community.

\section{RESULTS}

\section{Baseline characteristics}

Vaccination status was assessed for 4862 children aged 9-17 months contributing with 5956 observations (figure 2). In addition to the 2536 children not included as their vaccination status was not assessed, we excluded 18 children corresponding to 19 observations from the analyses. These were children with unknown date of MV 
Table 1 Baseline characteristics for observations of children (aged 9-17 months) included in the analyses by vaccination group

\begin{tabular}{|c|c|c|c|c|c|c|}
\hline & DTP<MV & $\mathrm{DTP}=\mathrm{MV}$ & DTP>MV & DTP, no MV & No DTP, no MV & $P$ value \\
\hline Numbers (\%) & $1590(27)$ & $1491(25)$ & $493(8)$ & $1816(31)$ & 547 (9) & \\
\hline Sex & & & & & & 0.29 \\
\hline Female (\%) & $756(48)$ & $762(51)$ & $238(48)$ & $885(49)$ & $257(47)$ & \\
\hline $\begin{array}{l}\text { Median age in } \\
\text { months at start of } \\
\text { follow-up (IQR) }\end{array}$ & $14.0(11.8-15.9)$ & $13.8(11.9-16.0)$ & $15.3(13.6-16.8)$ & $11.4(10.0-13.5)$ & $12.0(10.4-14.7)$ & $<0.0001$ \\
\hline $\begin{array}{l}\text { MUAC z-score at } \\
\text { start of follow-up }\end{array}$ & $-0.93(1.09)$ & $-1.09(1.05)$ & $-1.16(1.08)$ & $-1.09(1.13)$ & $-1.13(1.13)$ & $<0.0001$ \\
\hline Region & & & & & & $<0.0001$ \\
\hline Oio & 303 (19) & 337 (23) & $87(18)$ & $413(23)$ & $167(31)$ & \\
\hline Biombo & $405(25)$ & $283(19)$ & $108(22)$ & $386(21)$ & $147(27)$ & \\
\hline Ethnicity & & & & & & $<0.0001$ \\
\hline Balanta & $220(14)$ & $179(12)$ & $38(8)$ & $323(18)$ & 177 (33) & \\
\hline Pepel & $338(21)$ & $228(16)$ & $98(20)$ & $316(18)$ & $137(25)$ & \\
\hline Fula/Mandinca & $703(45)$ & $921(63)$ & $296(61)$ & $950(53)$ & $183(34)$ & \\
\hline Manjaco & $106(7)$ & $44(3)$ & $9(2)$ & $81(4)$ & $25(5)$ & \\
\hline Other & $208(13)$ & $96(7)$ & $45(9)$ & $134(7)$ & $18(3)$ & \\
\hline $\begin{array}{l}\text { Median maternal } \\
\text { age in years (IQR) }\end{array}$ & $25.6(20.6-30.8)$ & $26(21.2-30.6)$ & $25.9(21.3-31)$ & $26.2(20.8-30.9)$ & $26.8(21.3-31.5)$ & 0.05 \\
\hline $\begin{array}{l}\text { Education of } \\
\text { caretaker (in years) }\end{array}$ & & & & & & $<0.0001$ \\
\hline
\end{tabular}

Five hundred three observations with missing MUAC; 64 observations with missing information on ethnicity; 63 observations with missing information on maternal age; 110 observations with missing information on education of caretaker.

MUAC, mid-upper arm circumference; N/A, not available.

or DTP (8 children, 9 observations), and children who had received MV, but no DTP (10 children, 10 observations). We compared the distribution of baseline characteristics between children included in and excluded from the study (online supplementary table 1). Children excluded differed from the children included in the analyses with respect to age, region of residence, ethnicity and maternal age, but sex, nutritional status and maternal education did not differ. We also compared the distribution of baseline characteristics for different vaccination groups (table 1 ). The age of children differed by vaccination group: children with DTP $>$ MV were older than children who received DTP before or together with MV and children without MV were younger $(p<0.0001)$. Mean MUAC z-scores for all groups were around 1 SD below the reference, but children with DTP $>$ MV and no DTP, no MV tended to deviate more from the WHO reference curve for MUAC compared with the other groups. The distribution of vaccination groups differed by region and ethnicity. More mothers of children vaccinated out-of-sequence or with missing MV had never attended school than mothers of children vaccinated in-sequence. Children vaccinated out-of-sequence had received their most recent vaccine closer to entry in the analysis (table 1 ).

\section{Mortality by vaccination group among children aged 9-17} months

Children vaccinated out-of-sequence had higher mortality compared with children vaccinated in-sequence (MRR: 2.10, $95 \%$ CI 1.07 to 4.11 ; DTP=MV: $2.30,95 \%$ CI 1.15 to 


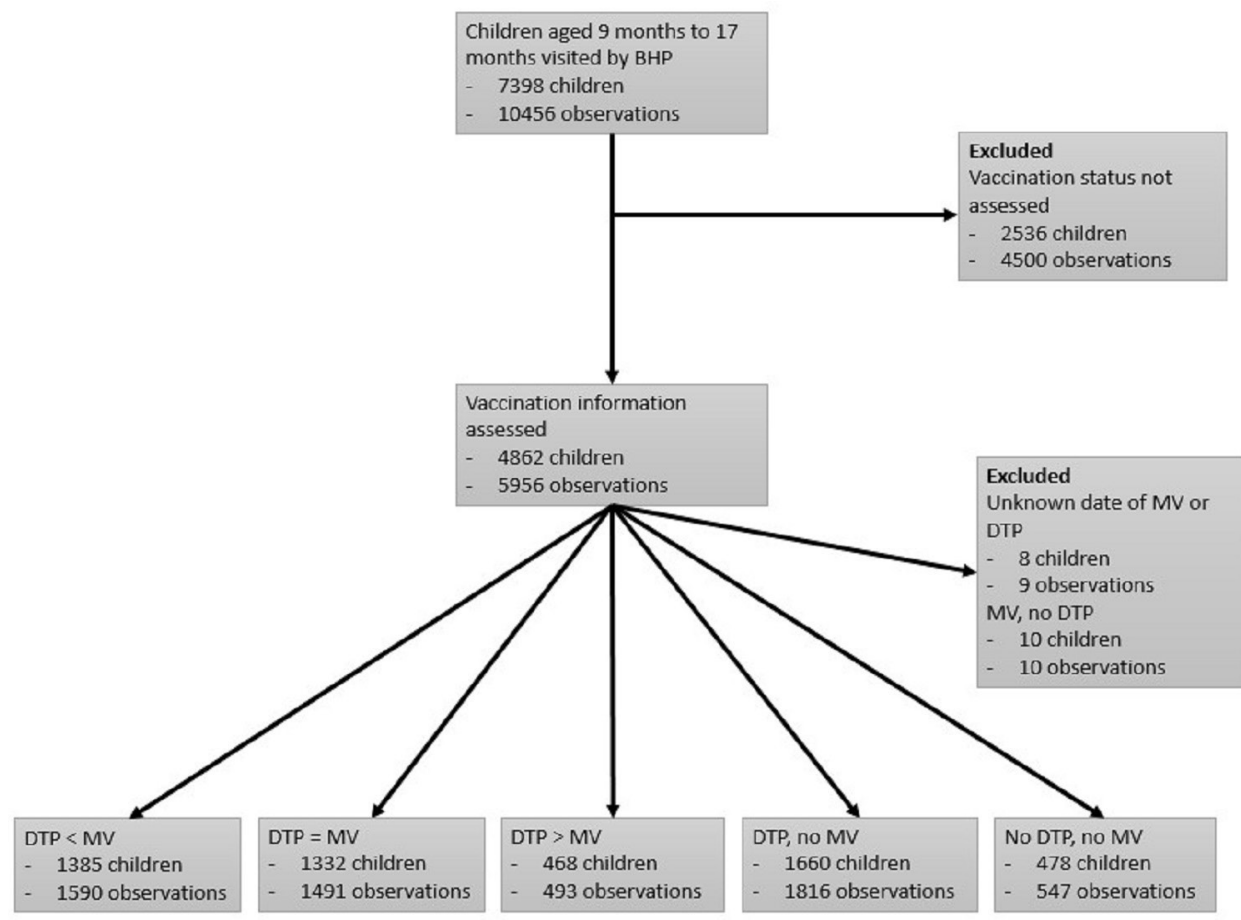

Figure 2 Flow chart of children included and excluded from the analysis. BHP, Bandim Health Project; DTP, diphtheriatetanus-pertussis vaccine; MV, measles vaccine.

4.58) and DTP $>$ MV: $1.45,95 \%$ CI 0.50 to 4.22$)$. Children who had received DTP, but no MV had higher mortality compared with children vaccinated in-sequence (MRR: $2.57,95 \%$ CI 1.37 to 4.83$)$. Children without DTP and MV had higher mortality than children vaccinated in-sequence (MRR: $3.04,95 \%$ CI 1.41 to 6.55 ) (table 2). The associations were similar for boys and girls $(\mathrm{p}=0.77)$. For boys, out-of-sequence vaccinations were associated with an MRR of 1.96 (95\% CI 0.80 to 4.78); for girls, the MRR was 2.25 (95\% CI 0.81 to 6.30 ). DTP without MV was associated with significantly higher mortality for boys (MRR: $3.41,95 \%$ CI 1.50 to 7.77 ); mortality for girls was also higher, but not statistically significant (MRR: 1.67, 95\% CI 0.62 to 4.50 ) (table 2).

We have previously estimated an MRR of 0.48 (95\% CI 0.27 to 0.87 ) for MV versus no MV, without taking sequence of vaccination into consideration. ${ }^{11}$ When we examined the NSEs of MV by comparing children MV-vaccinated in-sequence with children not MV-vaccinated, we found an MRR of 0.40 (95\% CI 0.23 to 0.69) (data not shown).

\section{Mortality by vaccination group among children aged $18-35$ months}

Initially, mortality declined with age as expected (figure 1). However, in spite of being older, in-sequence vaccinated children had higher mortality at 18-35 months of age (mortality rate (MR): 39.9 per 1000 person years (PYRS)) than children aged 9-17 months (MR: 32.6 per 1000 PYRS). Mortality developed differently with age for children vaccinated in-sequence compared with children vaccinated out-of-sequence (figure 3 ). Since the in-sequence group had high mortality, there was no real differences in mortality between out-of-sequence and in-sequence vaccinations in the 18-35 months age group (online supplementary table 2). The MRR for out-of-sequence compared with in-sequence vaccinated children differed significantly between the age group 9-17 months (table 2) and 18-35 months (online supplementary table 2) (test of interactions, $\mathrm{p}=0.02$ ).

\section{Sensitivity analyses}

In the age group 9-17 months at least $20 \%$ of children vaccinated out-of-sequence received further doses of DTP during follow-up, but few children vaccinated in-sequence did (online supplementary table 3 ). To minimise the effect of vaccinations during follow-up, we conducted two sensitivity analyses. First, we censored follow-up 2 months after entry since few additional vaccines would be provided in that time window. This clearly restricted the power, but the trends remained the same: out-of-sequence vaccinations were associated with an MRR of 2.51 (95\% CI 0.86 to 7.35 ) (table 3 ). The estimates changed more for girls; out-of-sequence vaccinations being associated with an eightfold higher mortality for girls (MRR: 7.83, 95\% CI 0.90 to 67.83 ). Second, we restricted the dataset to children who had received DTP3 and therefore were unlikely to receive additional routine DTP vaccinations during follow-up (online supplementary table 4). The MRR of out-of-sequence vaccinations compared with in-sequence vaccinations was 1.85 (95\% CI 0.82 to 4.16 ), and the effect was similar for boys and girls $(\mathrm{p}=0.60)$ (online supplementary table 4). For girls, both DTP $3=$ MV and DTP3 $>$ MV were associated with higher mortality. For boys, 


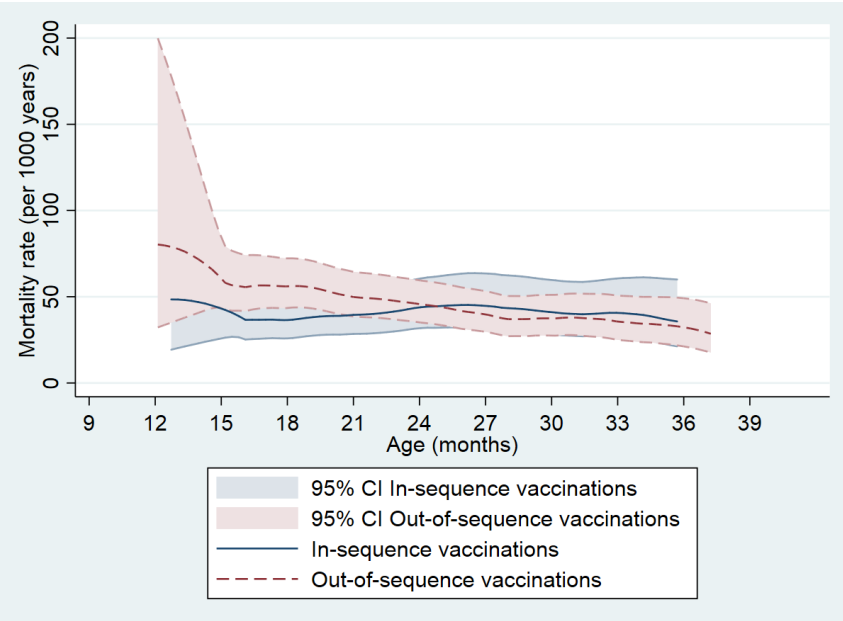

Figure 3 Mortality rate according to sequence of DTP and MV vaccinations among children visited between 9 and 35 months of age. The figure plots unadjusted mortality rates by vaccination status (in-sequence vs out-of-sequence vaccinations) among children with a vaccination card seen between 9 and 35 months of age. The smoothed mortality curve starts shortly before 12 months of age, where the first event occurs. Only few children contribute with observation time between 9 and 12 months. DTP, diphtheria-tetanuspertussis vaccine; MV, measles vaccine.

DTP3=MV were associated with higher mortality, whereas DTP3>MV was not (online supplementary table 4).

\section{DISCUSSION}

\section{Main findings}

Out-of-sequence vaccinations were associated with higher mortality compared with in-sequence vaccinations. After 18 months, the recommended age of booster DTP vaccination, the general mortality rate increased and the differential effect of out-of-sequence vaccinations disappeared.

\section{Strengths and weaknesses}

Using the landmark approach, survival bias was prevented since the vaccination status of the children was only updated when vaccination status was re-assessed, thereby preventing that vaccination information was updated for surviving children, but not for dead children. While this approach does not misclassify observation time dependent on the outcome, the misclassification of vaccinations during follow-up would yield conservative estimates. ${ }^{31}$

Data were collected through the rural HDSS in Guinea-Bissau and vaccination status was based on the vaccination card being inspected. Vaccinated children, whose vaccination card was not presented, were not included in the analysis. Mortality as the main outcome is unlikely to be reported wrongly, and with visits every 6 months, the imprecision in date of death is limited. Booster doses of DTP were not registered before 1996 and we could not fully explore the effect of booster DTP in the present cohort. To limit the effect of vaccinations during follow-up, we censored the main analysis at 18 months of age, when the 


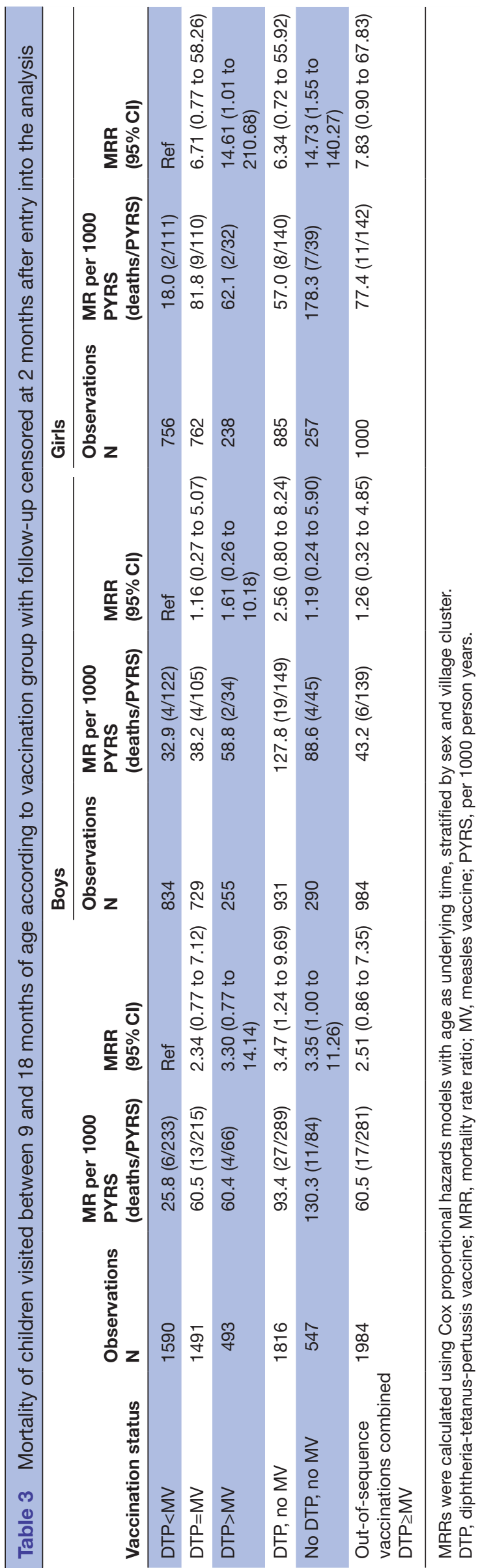

children were eligible for the DTP booster; furthermore, we conducted two sensitivity analyses in which we first restricted follow-up to 2 months after entry and second limited the analysis to children who had received three doses of DTP. The conclusions of the main analysis were robust in these sensitivity analyses. The statistical model used, only compared children within the same village cluster, thus limiting bias from local differences such as epidemics, ethnicity and access to healthcare. Comparing children across clusters did not change the conclusions (data not shown).

In spite of the careful collection of vaccination information and individual level follow-up, we cannot guarantee that observed mortality differences are caused only by the sequence of vaccinations. To limit confounding, we assessed whether available background factors changed the estimate by $>10 \%$. As no background factor changed the estimate by $>10 \%$, we did not present adjusted estimates. However, there may be residual confounding not adjusted for.

To enter the analysis, a child had to survive to have the vaccination card inspected, and a differential mortality pattern before the inspection of the vaccination card would not be captured in our analyses. However, in prior studies of vaccination sequence and mortality, the effects have been similar regardless of whether vaccinations are registered at the time of vaccinations ${ }^{2632}$ or later, ${ }^{24} 27$ and this is therefore unlikely to explain the pattern.

\section{Comparison with other studies}

Similar to our study, previous studies have found that out-of-sequence vaccinations are associated with increased mortality. ${ }^{24-26}$ 33-35 In the WHO-commissioned review, out-of-sequence vaccinations with DTP and MV were associated with a relative MR of 2.34 (95\% CI 1.57 to 3.50) compared with MV after DTP. ${ }^{7}$ Hence, the age group 9-17 months in the present study is entirely consistent with previous studies. Out-of-sequence vaccinations may affect mortality and hospital admissions; large population-based cohort studies from Denmark found that out-of-sequence vaccinations of DTP and MV were associated with higher hospitalisation rates. ${ }^{36} 37$ To our knowledge, no study without survival bias has found beneficial effects of out-of-sequence vaccinations with DTP and MV.

The original study assessed the effect of MV compared with no MV and found an MRR of 0.48 (95\% CI 0.27 to 0.87$)^{11}$ not accounting for sequence of vaccination. According to our analyses, this has underestimated the NSEs of MV. When we considered sequence of vaccination and compared children MV-vaccinated in-sequence with children not MV-vaccinated, we found an MRR of 0.40 (95\% CI 0.23 to 0.69 ).

The mortality rate usually declines with age. ${ }^{38}$ In our study, among children vaccinated in-sequence, we found higher mortality rate in children aged 18-35 months compared with children aged 9-17 months (figure 3). Since mortality did decline with age in the younger age group, we speculate that DTP booster for which children 
were eligible at 18 months of age may have contributed to this pattern just like DTP out-of-sequence with MV was associated with higher mortality. Unfortunately, our data collection tool in the early 1990 did not systematically assess DTP booster coverage. According to UNICEF figures, the DTP3 coverage was low in 1991-1996 (45\%$74 \%),{ }^{39}$ and we would not expect the coverage of booster DTP to be high. In urban Bissau, where the coverage for booster DTP was high, we have previously shown a similar increase in mortality after 18 months of age. ${ }^{5}$ Thus, DTP booster doses may partly explain the higher mortality among children aged 18-35 months, as observed in Gambia and India. ${ }^{55} 40$

Effects were similar for boys and girls, and overall we found no sex-differential effect of out-of-sequence vaccinations. However, other studies have found higher female mortality when DTP was administered after MV, ${ }^{21}{ }^{40}$ for example, high-titre measles vaccine (HTMV) was associated with higher female mortality and had to be withdrawn because most HTMV recipients had received DTP after MV. ${ }^{28}$ In the present cohort, few children had received DTP after MV and most out-of-sequence vaccinations were combined administration of DTP and MV. When follow-up was limited to 2 months, estimates for out-of-sequence changed more for girls than for boys even though the difference between boys and girls did not reach statistical significance.

\section{Interpretation and implications}

We found that out-of-sequence vaccinations were associated with higher mortality both for children with co-administration of DTP and MV, and children with DTP after $\mathrm{MV}$, compared with children vaccinated in-sequence. It could be speculated that out-of-sequence vaccinated children just had higher mortality because they were frail or their mothers less compliant with health services. In the present study, it speaks against the effect being due to an inherent bias that the difference disappeared completely for children aged 18-35 months, possibly due to booster DTP. Furthermore, evidence from RCTs of medium-titre MV and HTMV strongly supported that an inactivated vaccine after MV was associated with higher mortality. ${ }^{28}$ Thus, sequence of vaccinations is likely to be important for child survival and should be considered when planning, implementing and evaluating the childhood vaccination programmes.

Current vaccination recommendations are based merely on the disease-specific effects of vaccines, often based on surrogate measures of the ability to prevent targeted infections. However, if vaccines alter the susceptibility to other infections this should be considered. Currently, vaccination programmes are evaluated based on vaccination coverage of DTP and MV at 12 months of age, and timeliness or sequence of vaccination is not taken into account. We found that DTP not succeeded by MV was associated with increased mortality and that out-of-sequence vaccinations were associated with higher mortality compared with children vaccinated in-sequence, thus, the current evaluation criteria emphasising DTP3 coverage may not optimise the impact of the vaccination programme on child health. Our results indicate that a stronger emphasis should be put on increasing the MV coverage and getting DTPs and MV in the recommended sequence.

Currently, WHO is planning to introduce the second year of life platform with several inactivated vaccines (booster DTP, meningitis A, RTS,S malaria vaccine) ${ }^{29}$ Hence, in the future children may receive inactivated vaccines after live MV at 9 months of age, because they deviate from the recommended schedule, and if they follow the schedule. We urge others to test the effect of providing non-live vaccines after MV, preferably prior to the introduction of new vaccines, while RCTs are still possible.

\section{CONCLUSION}

Overall, we found that out-of-sequence vaccinations in children were associated with higher mortality compared with children vaccinated in-sequence. Vaccination programmes should monitor the sequence of vaccinations to optimise the overall effect on child survival.

Acknowledgements The authors would like to thank all children and mothers contributing with information to the present study. The authors would also like to thank the dedicated staff working at BHP in Guinea-Bissau for the great job they have done regarding data collection, data entry and data cleaning for the present study.

Contributors SMT, PA and ABF designed the study and planned the analyses. SMT extracted, cleaned and analysed the data. PA supervised the data collection and data entry. SMT drafted the paper with assistance from PA, AR and ABF. All authors read and approved the final manuscript.

Funding This work was supported by European Union FP7 support for OPTIMUNISE (Health-F3-2011-261375), and by DANIDA travel grant (grant no. A27607). The Bandim Health Project received support from the Danish National Research Foundation via Research Center for Vitamins and Vaccines (DNRF108).

Competing interests None declared.

Patient consent for publication Not required.

Provenance and peer review Not commissioned; externally peer reviewed. Data availability statement Data are available on reasonable request.

Open access This is an open access article distributed in accordance with the Creative Commons Attribution Non Commercial (CC BY-NC 4.0) license, which permits others to distribute, remix, adapt, build upon this work non-commercially, and license their derivative works on different terms, provided the original work is properly cited, appropriate credit is given, any changes made indicated, and the use is non-commercial. See: http://creativecommons.org/licenses/by-nc/4.0/.

\section{REFERENCES}

1. Liu L, Oza S, Hogan D, et al. Global, regional, and national causes of under-5 mortality in 2000-15: an updated systematic analysis with implications for the sustainable development goals. Lancet 2016;388:3027-35.

2. Feikin DR, Flannery B, Hamel MJ, et al. Vaccines for children in Low- and Middle-Income Countries. In: Black RE, Laxminarayan R, Temmerman M, et al, eds. Reproductive, maternal, newborn, and child health: disease control priorities: world bank, 2016.

3. Plotkin S. History of vaccination. Proc Natl Acad Sci U S A 2014;111:12283-7

4. Benn CS, Netea MG, Selin LK, et al. A small jab - a big effect: nonspecific immunomodulation by vaccines. Trends Immunol 2013;34:431-9. 
5. Aaby P, Kollmann TR, Benn CS. Nonspecific effects of neonatal and infant vaccination: public-health, immunological and conceptual challenges. Nat Immunol 2014;15:895-9.

6. Aaby P, Benn CS. Non-Specific and sex-differential effects of routine vaccines: what evidence is needed to take these effects into consideration in low-income countries? Hum Vaccin 2011;7:120-4.

7. Higgins JPT, Soares-Weiser K, López-López JA, et al. Association of BCG, DTP, and measles containing vaccines with childhood mortality: systematic review. BMJ 2016;355.

8. Aaby P, Samb B, Simondon F, et al. Non-Specific beneficial effect of measles immunisation: analysis of mortality studies from developing countries. BMJ 1995;311:481-5.

9. Desgrées du Loû A, Pison G, Aaby P. Role of immunizations in the recent decline in childhood mortality and the changes in the female/male mortality ratio in rural Senegal. Am J Epidemiol 1995;142:643-52.

10. Aaby P, Bhuiya A, Nahar $L$, et al. The survival benefit of measles immunization may not be explained entirely by the prevention of measles disease: a community study from rural Bangladesh. Int $J$ Epidemiol 2003;32:106-15.

11. Kristensen I, Aaby P, Jensen $H$. Routine vaccinations and child survival: follow up study in Guinea-Bissau, West Africa. BMJ 2000;321:1435-8.

12. Fisker $A B$, Hornshøj $L$, Rodrigues $A$, et al. Effects of the introduction of new vaccines in Guinea-Bissau on vaccine coverage, vaccine timeliness, and child survival: an observational study. Lancet Glob Health 2014;2:e478-87.

13. Aaby P, Martins CL, Garly M-L, et al. Non-Specific effects of standard measles vaccine at 4.5 and 9 months of age on childhood mortality: randomised controlled trial. BMJ 2010;341:c6495.

14. World Health Organization. Summary of the SAGE April 2014 meeting, 2014. Available: http://www.who.int/immunization/sage/ meetings/2014/april/report_summary_april_2014/en/ [Accessed 10 Apr 2014].

15. Aaby P, Jensen H, Gomes J, et al. The introduction of diphtheriatetanus-pertussis vaccine and child mortality in rural Guinea-Bissau: an observational study. Int J Epidemiol 2004;33:374-80.

16. Mogensen SW, Andersen A, Rodrigues A, et al. The introduction of diphtheria-tetanus-pertussis and oral polio vaccine among young infants in an urban African community: a natural experiment. EBioMedicine 2017;17:192-8.

17. Aaby P, Mogensen SW, Rodrigues A, et al. Evidence of Increase in Mortality After the Introduction of Diphtheria-Tetanus-Pertussis Vaccine to Children Aged 6-35 Months in Guinea-Bissau: A Time for Reflection? Front Public Health 2018;6.

18. Welaga P, Nielsen J, Adjuik M, et al. Non-Specific effects of diphtheria-tetanus-pertussis and measles vaccinations? an analysis of surveillance data from Navrongo, Ghana. Trop Med Int Health 2012;17:1492-505

19. Agergaard J, Nante E, Poulstrup G, et al. Diphtheria-TetanusPertussis vaccine administered simultaneously with measles vaccine is associated with increased morbidity and poor growth in girls. A randomised trial from Guinea-Bissau. Vaccine 2011;29:487-500.

20. Aaby P, Benn C, Nielsen J, et al. Testing the hypothesis that diphtheria-tetanus-pertussis vaccine has negative non-specific and sex-differential effects on child survival in high-mortality countries. BMJ Open 2012;2:e000707.

21. Aaby P, Garly M-L, Nielsen J, et al. Increased female-male mortality ratio associated with inactivated polio and diphtheria-tetanuspertussis vaccines: observations from vaccination trials in GuineaBissau. Pediatr Infect Dis J 2007;26:247-52.

22. Aaby P, Ravn H, Benn CS. The who review of the possible nonspecific effects of diphtheria-tetanus-pertussis vaccine. Pediatr Infect Dis J 2016;35:1247-57.
23. Benn CS, Aaby P. Diphtheria-Tetanus-Pertussis vaccination administered after measles vaccine: increased female mortality? Pediatr Infect Dis J 2012;31:1095-7.

24. Aaby $\mathrm{P}$, Biai $\mathrm{S}$, Veirum JE, et al. Dtp with or after measles vaccination is associated with increased in-hospital mortality in Guinea-Bissau. Vaccine 2007;25:1265-9.

25. Aaby P, Jensen H, Samb B, et al. Differences in female-male mortality after high-titre measles vaccine and association with subsequent vaccination with diphtheria-tetanus-pertussis and inactivated poliovirus: reanalysis of West African studies. Lancet 2003;361:2183-8.

26. Fisker $\mathrm{AB}$, Ravn $\mathrm{H}$, Rodrigues $\mathrm{A}$, et al. Co-Administration of live measles and yellow fever vaccines and inactivated pentavalent vaccines is associated with increased mortality compared with measles and yellow fever vaccines only. An observational study from Guinea-Bissau. Vaccine 2014;32:598-605.

27. Welaga P, Oduro A, Debpuur C, et al. Fewer out-of-sequence vaccinations and reduction of child mortality in northern Ghana. Vaccine 2017;35:2496-503.

28. Aaby P, Ravn H, Benn CS, et al. Randomized trials comparing inactivated vaccine after medium- or high-titer measles vaccine with standard titer measles vaccine after inactivated vaccine: a metaanalysis. Pediatr Infect Dis J 2016;35:1232-41.

29. Anon. Meeting of the strategic Advisory group of experts on immunization, April 2016 - conclusions and recommendations. Wkly Epidemiol Rec 2016;91:266-84.

30. World Health Organization. Multicentre growth reference Study Group. who child growth standards: Length/height-for-age, weight-for-age, weight-for-length, weight-for-height and body mass index-for-age: methods and development. Geneva: World Health Organisation, 2006.

31. Jensen H, Benn CS, Lisse IM, et al. Survival bias in observational studies of the impact of routine immunizations on childhood survival. Trop Med Int Health 2007;12:5-14.

32. Fisker AB, Thysen SM. Non-live pentavalent vaccines after live measles vaccine may increase mortality. Vaccine 2018;36:6039-42.

33. Aaby P, Ibrahim SA, Libman MD, et al. The sequence of vaccinations and increased female mortality after high-titre measles vaccine: trials from rural Sudan and Kinshasa. Vaccine 2006;24:2764-71.

34. Aaby P, Jensen $\mathrm{H}$, Walraven G. Age-specific changes in the female-male mortality ratio related to the pattern of vaccinations: an observational study from rural Gambia. Vaccine 2006;24:4701-8.

35. Hirve S, Bavdekar A, Juvekar S, et al. Non-Specific and sexdifferential effects of vaccinations on child survival in rural Western India. Vaccine 2012;30:7300-8.

36. Sørup S, Benn CS, Poulsen A, et al. Live vaccine against measles, mumps, and rubella and the risk of hospital admissions for nontargeted infections. JAMA 2014;311:826-35.

37. Sørup S, Benn CS, Poulsen A, et al. Simultaneous vaccination with MMR and DTaP-IPV-Hib and rate of hospital admissions with any infections: a nationwide register based cohort study. Vaccine 2016;34:6172-80.

38. Liu L, Hill K, Oza S, et al. Levels and Causes of Mortality under Age Five Years. In: Black RE, Laxminarayan R, Temmerman M, et al, eds. Reproductive, maternal, newborn, and child health: disease control priorities: world bank, 2016

39. UNICEF. Immunization, 2016. Available: http://data.unicef.org/topic/ child-health/immunization/ [Accessed 29 Sep 2016].

40. Krishnan A, Srivastava R, Dwivedi P, et al. Non-specific sexdifferential effect of DTP vaccination may partially explain the excess girl child mortality in Ballabgarh, India. Trop Med Int Health 2013;18:1329-37. 